\title{
Is deep brain stimulation (DBS) a safe and effective treatment for adults with dystonia? - A Cochrane Review summary with commentary
}

\author{
Francesca Cecchi \\ Fondazione don Carlo Gnocchi Scientific Institute \\ Fondazione don Carlo Gnocchi, Via di Scandicci 269, 50143, Firenze, Italy \\ Tel.: +39055 73931; E-mail: fcecchi@dongnocchi.it
}

\begin{abstract}
.
BACKGROUND: Dystonia is the third common motor disorder; deep brain stimulation (DBS) has been reported as beneficial in selected dystonia patients.

OBJECTIVE: To assess the effects of DBS in adults with dystonia.

METHODS: Cochrane Review conducted by Rodrigues et al. searching for RCTs with a parallel design, comparing DBS versus placebo, sham intervention, or best medical/surgical treatment was summarized and discussed from a rehabilitation perspective.

RESULTS: Only two RCTs, at high risk of bias, and comparing DBS with sham intervention, were selected; low quality evidence was found that DBS may improve specific symptoms and functionality in adults with cervical or segmental/generalized dystonia; no conclusion was reached on DBS safety and tolerability.

CONCLUSIONS: High quality studies are needed to verify DBS effects in dystonia; more types of dystonia and other control interventions also need to be addressed.
\end{abstract}

Keywords: Dystonia, deep brain stimulation

The aim of this commentary is to discuss in a rehabilitation perspective the recently published Cochrane Review "Deep brain stimulation for dystonia", by Filipe B Rodrigues, Gonçalo S Duarte, David Prescott, Joaquim Ferreira, João Costa ${ }^{1}$, under

\footnotetext{
${ }^{1}$ The abstract/plain language summary of this Cochrane Review is taken from a Cochrane Review previously published in the Cochrane Database of Systematic Reviews Reviews
}

the direct supervision of Cochrane Movement Disorders Group. This Cochrane Corner is produced

2019, Issue 1. Art. No.: CD012405, DOI: 10.1002/14651858. CD012405.pub2 (see www.cochranelibrary.com for information). Cochrane Reviews are regularly updated as new evidence emerges and in response to feedback, and Cochrane Database of Systematic Reviews should be consulted for the most recent version of the review. 
in agreement with NeuroRehabilitation by Cochrane Rehabilitation.

\section{Background}

After Parkinson's disease and essential tremor, dystonia is the third most common movement disorder, describing a spectrum of conditions characterized by sustained or intermittent involuntary muscle contractions, causing abnormal, often repetitive, movements or postures.

Botulinum toxin is recommended as first-line treatment for focal dystonia, but its efficacy is variable and may wane over time; the pharmacological treatment of generalized dystonia is more challenging, bearing poor results (Cloud and Jinnah, 2010).

Deep brain stimulation (DBS) applies an electrical current to specific subcortical nuclei, through surgically implanted electrodes, wire-connected to a subcutaneously-placed pulse generator; tipically, the internal globus pallidus is the primary target in dystonia.

Recent studies reported the beneficial effects of DBS in dystonia, as in selected cases of Parkinson's disease and in essential tremor, but serious events have also been reported, and no systematic review had yet addressed the outcomes of DBS in patients with dystonia.

Deep brain stimulation for dystonia

Filipe B Rodrigues, Gonçalo S Duarte, David Prescott, Joaquim Ferreira, João Costa, 2019.

\section{Objective}

The aim of this Cochrane Review was to assess the effects of DBS versus placebo, sham intervention, or best medical or surgical (resective/lesional surgery) care, on adult patients with dystonia, in terms of efficacy, safety and tolerability.

\section{What was studied and methods}

Adult (age $>18$ ) patients with clinical diagnosis of any type of dystonia were studied. RCTs with a parallel design were considered, comparing DBS versus placebo, sham intervention, or best medical/surgical treatment, for: dystonia-specific symptoms, adverse events, clinical status, quality of life (QoL), functional capacity, mood and tolerability.

\section{Results}

Only two RCTs, both attributed high risk of bias, were included, comparing DBS of the internal globus pallidus nucleus with sham stimulation, in adults with generalized or segmental dystonia (2006), or focal cervical dystonia (2014), enrolling a total of 102 participants, 54 men, assessing outcomes at 3 months (stimulation started also in controls) and 9 months from surgery.

As the body distribution of dystonia was different, efficacy was assessed separately for each study.

In segmental/ generalized dystonia, results show that DBS may improve:

- Specific symptoms (MD 14.4 units, 95\% CI 8.0 to 20.8 ; 1 RCT, 40 participants, low-quality evidence)

- Clinical status (MD 3.5 units, 95\% CI 2.33 to 4.67;1 RCT, 37 participants, low-quality evidence)

- Physical functioning-related QoL (MD 6.3 units, 95\% CI 1.06 to 11.54 ; 1 RCT, 33 participants, low-quality evidence),

- Dystonia-related functional capacity at three months (MD 3.1 units, 95\% CI 1.71 to $4.48 ; 1$ RCT, 39 participants, low-quality evidence).

It is uncertain whether DBS improves physical functioning-related QoL (MD 5.0 units, 95\% CI -2.14 to $12.14,1$ RCT, 33 participants; very lowquality evidence), or mental health-related QoL (MD -4.6 units, $95 \%$ CI -11.26 to 2.06 ; 1 RCT, 30 participants; very low-quality evidence).

In cervical dystonia, DBS may improve:

- Cervical dystonia-related symptoms (mean difference (MD) 9.8 units, $95 \%$ CI 3.52 to 16.08 units; 1 RCT, 59 participants, low-quality evidence),

- Cervical dystonia-related functional capacity (MD 3.8 units, 95\% CI 1.41 to 6.19 ; 1 RCT, 61 participants, low quality evidence)

- Mood at three months (MD 3.1 units, 95\% CI 0.73 to 5.47 ; 1 RCT, 61 participants, low-quality evidence).

- Clinical status, small effect size (MD 2.3 units, 95\% CI 1.15 to 3.45 ; 1 RCT, 61 participants, low-quality evidence).

It is uncertain whether DBS improves QoL (MD 3 units, 95\% CI -7.71 to 13.71 ; 1 RCT, 57 participants; very low-quality evidence), or emotional state 
(MD 2.4 units, 95\% CI -6.2 to $11.00 ; 1$ RCT, 56 participants; very low-quality evidence).

Safety and tolerability pooled results (as both DBS and sham intervention were similar) are inconclusive for either risk of adverse events (relative risk (RR) $1.58,95 \% 0.98$ to $2.54 ; 2$ RCTs, 102 participants, very low quality evidence), or tolerability (RR 1.86, 95\% CI 0.16 to $21.57 ; 2$ RCTs, 102 participants, very low quality evidence).

\section{Conclusions}

The Authors concluded that there is low quality evidence that DBS of the internal globus pallidus nucleus may improve dystonia-specific symptoms and functionality in adults with cervical or segmental/generalized dystonia; in the latter, DBS may also improve QoL; there is uncertainty as to DBS-related QoL improvement in cervical dystonia, and as to DBS safety and tolerability.

\section{Implications for practice in neurorehabilitation}

This first systematic review found only low quality evidence on DBS efficacy in adults with cervical and in segmental/generalized dystonia, not generalizable to other dystonia populations, and unverified in the long term; DBS was compared with sham surgery, and no conclusion was reached on DBS safety and tol- erability. Thus, although DBS remains the preferred surgical treatment for patients with severe, drugrefractory, isolated dystonia, its risk/benefit ratio is far from being established. Further research is needed on different dystonia populations, devices, stimulation protocols, and targeted nuclei, and on DBS long-term effects. Low quality evidence of DBS improving functionality of patients with dystonia was reported, but no implication regarding rehabilitation was addressed.

\section{Conflict of interest}

The author declares no conflicts of interest.

\section{Acknowledgments}

The author thanks Cochrane Rehabilitation and Cochrane Movement Disorders Group for reviewing the contents of the Cochrane Corner.

\section{References}

Cloud, L. J., \& Jinnah, H. A. (2010). Treatment strategies for dystonia. Expert Opin Pharmacother, 11(1), 5-15. doi: 10.1517/ 14656560903426171.

Rodrigues, F. B., Duarte, G. S., Prescott, D., Ferreira, J., \& Costa, J. (2019). Deep brain stimulation for dystonia. Cochrane Database of Systematic Reviews, 1, CD012405. https://doi.org/ 10.1002/14651858.CD012405.pub2. 